\title{
Increased risk of pernicious anemia following scabies: a nationwide population-based matched-cohort study
}

This article was published in the following Dove Press journal:

Therapeutics and Clinical Risk Management

13 September 2017

Number of times this article has been viewed

Jui-Ming Liu, ${ }^{1-3, *}$ Ren-Jun Hsu, ${ }^{3-5, *}$ Fung-Wei Chang, ${ }^{6}$ Feng-Hsiang Chiu, ${ }^{7,8}$ Chia-Lun Yeh,' Chun-Fa Huang, , 10 Shu-Ting Chang," Hung-Chang Lee, ${ }^{12} \mathrm{Hsin} \mathrm{Chi},{ }^{12,13}$ Chien-Yu Lin ${ }^{14,15}$

'Division of Urology, Department of Surgery, Taoyuan General Hospital, Ministry of Health and Welfare, Taoyuan, ${ }^{2}$ Department of Medicine, National Yang-Ming University, ${ }^{3}$ Graduate Institute of Life Sciences, ${ }^{4}$ Department of Pathology and Graduate Institute of Pathology and Parasitology, the Tri-Service General Hospital, National Defense Medical Center, ${ }^{5}$ Biobank Management Center of the Tri-Service General Hospital, ${ }^{6}$ Department of Obstetrics and Gynecology, Tri-Service General Hospital, National Defense Medical Center, Taipei, ${ }^{7}$ Department of Emergency Medicine, Shuang Ho Hospital, Taipei Medical University, New Taipei

City, Taiwan; ${ }^{8}$ Superintendent office, Lihuili Eastern Hospital, Ningbo, Zhejiang province, People's Republic of China; 'School of Chinese Medicine, College of Chinese Medicine, China Medical University, ${ }^{10}$ Department of Nursing, College of Medical and Health Science, Asia University, Taichung City, "Department of Gastroenterology, BinKun Women's and Children's Hospital, Taoyuan City, ${ }^{12}$ Department of Infectious Disease, MacKay Children's Hospital, Taipei, ${ }^{13}$ Department of Medicine, MacKay Medical College, New Taipe city, ${ }^{14}$ Department of Pediatrics and Infectious Disease, Hsinchu MacKay Memorial Hospital, Hsinchu City, Taiwan; ${ }^{15}$ Division of Infection and Pathway Medicine, College of Medicine and Veterinary Medicine, The University of Edinburgh, Scotland, UK

*These authors contributed equally to this work

Correspondence: Chien-Yu Lin Department of Pediatrics, Hsinchu MacKay Memorial Hospital, No 690, Sec 2, Guangfu Road, Hsinchu 300, Taiwan

Tel +88636119595

Fax +88636110900

Email mmhped.lin@gmail.com
Objectives: Scabies is a common and annoying disorder. Pernicious anemia (PA) is a serious disease which, when untreated, leads to death. Mounting evidence suggests that immunemediated inflammatory processes play a role in the pathophysiology of both diseases. The relationship between these two diseases has not been investigated. We conducted this study to explore the potential relationship between scabies and PA.

Materials and methods: This nationwide, population-based study was conducted using the National Health Insurance Research Database of Taiwan. In total, 5,407 patients with scabies were identified as a study group and 20,089 matched patients were randomly selected as a control group. We tracked patients in both groups for a 7-year period to identify the incidence of PA. The demographic characteristics and comorbidities of the patients were analyzed, and Cox proportional hazards regression was used to calculate the hazard ratios for PA.

Results: Of the 25,496 patients in this study, 183 (0.7\%) patients with newly diagnosed PA were identified during the 7-year follow-up period; 71 of 5,407 (1.3\%) from the scabies group and 112 of $20,089(0.6 \%)$ from the control group. Patients with scabies had a higher risk of subsequent PA, with a crude hazard ratio of 2.368. After adjusting for covariates, the adjusted hazard ratio was 1.51 (95\% confidence interval: 1.09-2.08).

Conclusion: This study demonstrated an increased risk of PA (adjusted hazard ratio 1.51) among patients with scabies. Immune-mediated inflammatory processes may contribute to this association. Further studies are warranted to investigate the entire pathological mechanisms between these two diseases. Physicians should pay attention to patients with history of scabies presented with anemia. Further confirmative tests of PA may contribute to correct diagnosis and initiation of vitamin B12 supplement.

Keywords: scabies, pernicious anemia, National Health Insurance Research Database, autoimmune gastritis, vitamin B12 deficiency, cobalamin

\section{Introduction}

Pernicious anemia (PA) is characterized by megaloblastic anemia caused by immunemediated chronic atrophic gastritis. ${ }^{1}$ Parietal cell destruction and autoantibodies against intrinsic factor result in malabsorption of vitamin B12, which is required for DNA synthesis, and megaloblastic anemia follows. The clinical manifestations of PA are nonspecific, but the presence of anti-intrinsic factor antibodies is highly confirmatory. In patients older than 60 years, the estimated prevalence of PA was $1.9 \%$, and it is believed that the condition is underdiagnosed. ${ }^{2}$ Immune-mediated inflammatory processes play a crucial role in the pathophysiology of PA, and 
an increased risk of several kinds of cancers is noted in patients with this condition. For example, a populationbased, case-control study of the US elderly population revealed an increased risk of developing noncardia gastric adenocarcinoma, gastric carcinoid tumors, tonsillar cancer, hypopharyngeal cancer, esophageal squamous cell carcinoma, small intestinal cancer, liver cancer, myeloma, acute myeloid leukemia, and myelodysplastic syndrome in patients with $\mathrm{PA}^{3}$

Scabies is a common infectious disorder, with a prevalence ranging from $0.2 \%$ to $71.4 \%$ of the general population in different countries, with the lowest prevalence in Iraq and highest in Papua New Guinea. ${ }^{4}$ The reported prevalence is greater than $10 \%$ in most countries and most prevalent in the Pacific and Latin American regions. Infestation by the mite Sarcoptes scabiei var. hominis causes intractable pruritus, and the disease burden is heavy. ${ }^{5}$ Scabies is more prevalent in patients who live in crowded environments and those with lower socioeconomic status. Children are infected most commonly with a peak occurring at 5-9 years. There are concerns about the long-term impacts of scabies. Infection of scabies will increase the risk of Streptococcus pyogenes infection, and a higher risk of long-term chronic kidney disease (CKD) was observed. ${ }^{6}$ Infection induces host immune reactions and systemic inflammatory processes are observed. Cytokine alterations are noted in patients with scabies, ${ }^{7,8}$ especially elevation of interleukin (IL)-17. Moreover, patients with scabies have higher risks of chronic obstructive pulmonary disease (COPD) and autoimmune diseases. Immune-mediated inflammatory cascades may contribute to the underpinning pathophysiology. ${ }^{9,10}$ Although systemic immune-mediated inflammatory processes are part of the pathophysiology of scabies, the whole picture of the long-term impact of scabies remains largely unclear.

Some conditions are more prevalent in patients with lower socioeconomic status, such as malnutrition, vitamin deficiency, and parasite infection. ${ }^{11}$ Both scabies and anemia are more common in this population. In addition, immune-mediated inflammatory processes play a role in the pathophysiologies of both scabies and PA. There is a possible link between these two diseases. We, therefore, conducted a nationwide population-based study to investigate the association between scabies and PA.

\section{Materials and methods Database}

This nationwide population-based cohort study was conducted using the National Health Insurance Research Database (NHIRD) of Taiwan. The National Health Insurance
(NHI) program is a unique public health and medical insurance system, which covered $99.9 \%$ of the 23 million residents of Taiwan as of the end of 2013. ${ }^{12,13}$ Various types of patient data, including demographic data, medical records, prescription records, and details of medical procedures, are recorded in the NHIRD. The Longitudinal Health Insurance Database 2000 (LHID2000) is a sub-dataset of the NHIRD, comprising data from one million people randomly selected from the larger NHIRD in 2000, and this was used for the analysis in our study. ${ }^{14}$

\section{Study population}

The patients included in the study population were chosen from the LHID2000 between January 2000 and December 2013. The clinical diagnoses of the patients were based on the International Classification of Diseases, 9th revision, Clinical Modification (ICD-9-CM) system. ${ }^{15}$ The diagnoses of scabies (ICD-9-CM: 133.0) and PA (ICD-9-CM: 281.0) were determined by licensed doctors. Patients newly diagnosed with scabies between January 2001 and December 2006 were enrolled as the study group (Figure 1). In total, 7,288 patients with scabies were identified; the following were excluded from the study: 1) patients who were diagnosed with scabies before January 1, $2001(\mathrm{n}=33)$; 2) patients with incomplete medical records $(\mathrm{n}=402) ; 3)$ patients who were younger than 20 years $(n=1,398)$; and 4) patients who had a previous history of scabies or PA $(n=48)$. Finally, 5,407 patients with scabies infection were enrolled as the scabies group. The index year referred to the year the patient was enrolled into the study. A matched cohort without scabies was enrolled by randomly matching patients by gender, age, insured region, and urbanization to the scabies group with a ratio of 1:4. In total, 20,089 subjects were enrolled in the non-scabies control group. Both cohorts were tracked for a 7-year period. The primary outcome of the study was to compare the incidence of PA in both groups. For each subject, the occurrence of PA was recorded for 7 years from the index year. In addition, we identified and analyzed the impact of gender, age, income, geography, urbanization, and comorbid diseases.

The study was approved by the Institutional Review Board of the Tri-Service General Hospital (approval number: TSGHIRB No B-105-06.). As this was a retrospective study and all data were anonymized, the Institutional Review Board committee agreed with the authors that it was not necessary to obtain patient consent. The statistical methods and results were reviewed by Chia-Lun Yeh, Institute of Bioinformatics and System Biology, National Chiao Tung University, Hsinchu, Taiwan. 


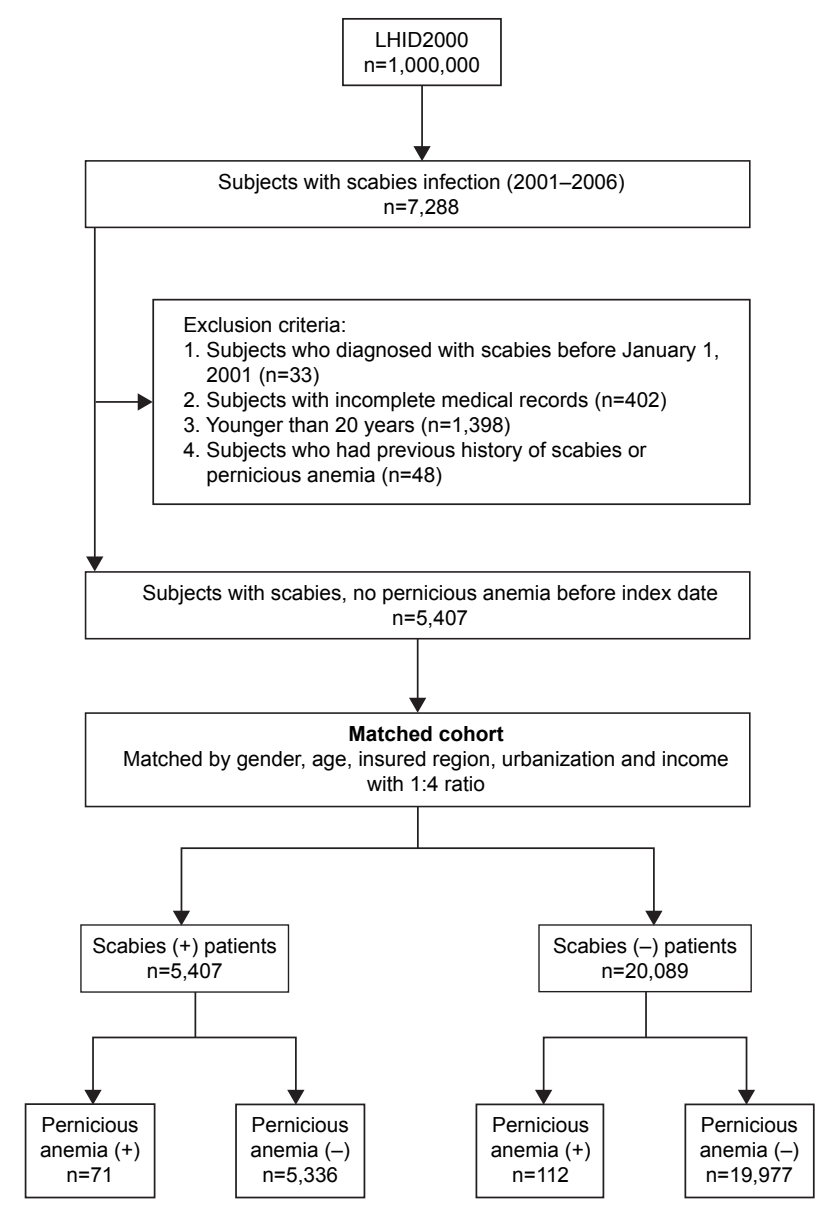

Figure I Flowchart of recruitment of subjects from the one-million random sample of the National Health Insurance Research Database from 2000 to 2006 in Taiwan. Notes: The exclusion criteria of the study is including (I) patients who were diagnosed with scabies before January I, 200I ( $\mathrm{n}=33)$, (2) patients with missing medical records $(n=402)$, (3) patients who were younger than 20 years $(n=1,398)$, (4) patients with history of scabies or pernicious anemia $(n=48)$.

Abbreviation: LHID, Longitudinal Health Insurance Database.

\section{Covariates}

The covariates of comorbidities that were selected in this study comprised diabetes mellitus (DM; ICD-9-CM: 250), hypertension (ICD-9-CM: 401-405), hyperlipidemia (ICD9-CM: 272.4), coronary heart disease (CHD; ICD-9-CM: 410-414), CKD (ICD-9-CM: 585, 586, 588), chronic liver disease (ICD-9-CM: 571), cerebral vascular accident (ICD9-CM: 430-438), and COPD (ICD-9-CM: 496). Participants were stratified by age into six groups of 10 -year intervals: 20-29 years, 30-39 years, 40-49 years, 50-59 years, 60-69 years, and older than 70 years. Monthly income in New Taiwan Dollars (NTD) was divided into four categories: less than NTD 20,000; NTD 20,000 to NTD 39,999; NTD 40,000 to NTD 59,999; and more than NTD 60,000. The geographic areas of residency in Taiwan were divided into four regions: the northern region, the central region, the southern region, and "other regions" (ie, eastern and outlying islands). Level of urbanization was classified into four groups.

\section{Statistical analysis}

SPSS software version 19.0 (SPSS Inc., Chicago, IL, USA) was used for statistical analysis, and Microsoft ${ }^{\circledR}$ SQL Server ${ }^{\circledR}$ 2008 software was used for data management. The chisquare test was used to analyze descriptive data including demographic characteristics, age, income, geography, level of urbanization, and comorbidities between the scabies group and the non-scabies control group. Cox proportional hazards regression models were used to estimate the effects of risk factors on the hazard ratios (HRs) with accompanying 95\% confidence intervals (CIs). All models were adjusted for the covariates (gender, age, income, geography, urbanization, and comorbidities). A two-sided $p<0.05$ was set as the determinant of statistical significance.

\section{Results}

In total, 25,496 patients were enrolled in the study; 5,407 in the scabies cohort and 20,089 in the non-scabies matched control cohort. Table 1 shows the demographic and medical characteristics of both groups. There were no differences in the prevalence of scabies infection among the gender or age groups. Most of the patients belonged to the lower income group $(p<0.05)$ and were residents of northern Taiwan $(p<0.001)$, which was the area with the highest population density in Taiwan. Hypertension, COPD, cerebral vascular accident, and DM were the most prevalent comorbidities. Patients with scabies had a higher prevalence of comorbid diseases.

During the 7-year follow-up period, 183 (0.7\%) patients with newly diagnosed PA were identified -71 of 5,407 (1.3\%) from the scabies group and 112 of 20,089 (0.6\%) from the control group. The incidence rates of PA between the scabies and control groups were significantly different. Patients with scabies had a higher risk of subsequent PA, with a crude HR of 2.368 (95\% CI: 1.759-3.188). The KaplanMeier survival curve for the cumulative incidences of PA of both groups is presented in Figure 2. Further Cox regression risk analysis was performed to analyze the risks of independent factors and the results are summarized in Table 2. Patients with scabies had an increased risk of subsequent PA, with an adjusted HR of 1.51 (95\% CI: 1.09-2.08). PA was also prevalent in women and elders (adjusted HRs 0.53 for men and 2.22 for patients $\geq 70$ years). The risk of PA was not significantly different among patients with different incomes, geographic residences, or levels of urbanization. Higher risks of PA were observed in patients with DM, CKD, and COPD (adjusted HRs: 1.6 for DM, 2.55 for CKD, and 1.57 for COPD). 
Table I Demographic data in both scabies and control groups

\begin{tabular}{|c|c|c|c|}
\hline \multirow[t]{2}{*}{ Variables } & $\begin{array}{l}\text { Scabies } \\
\text { n (\%) }\end{array}$ & $\begin{array}{l}\text { Controls } \\
\text { n (\%) }\end{array}$ & \multirow[t]{2}{*}{$p$-value } \\
\hline & $5,407(100 \%)$ & $20,089(100 \%)$ & \\
\hline Gender & & & 0.777 \\
\hline Female & 2,343 (43.3\%) & $8,662(43.1 \%)$ & \\
\hline Male & $3,064(56.7 \%)$ & II,427 (56.9\%) & \\
\hline Age & & & 0.633 \\
\hline $20-29$ & $829(15.3 \%)$ & 3,465 (I7.2\%) & \\
\hline $30-39$ & $657(12.2 \%)$ & 2,485 (I2.4\%) & \\
\hline $40-49$ & 735 (I3.6\%) & $2,776(13.8 \%)$ & \\
\hline $50-59$ & $661(12.2 \%)$ & $2,45 \mathrm{I}(12.2 \%)$ & \\
\hline $60-69$ & $611(11.3 \%)$ & 2,267 (II.3\%) & \\
\hline$\geq 70$ & I,9|4 (35.4\%) & $6,645(33.1 \%)$ & \\
\hline Income group NTDa & & & $<0.05^{*}$ \\
\hline$<20,000$ & 4,504 (83.3\%) & I7,048 (84.9\%) & \\
\hline $20,000-39,999$ & $564(10.4 \%)$ & $2,020(10.1 \%)$ & \\
\hline $40,000-59,999$ & 257 (4.8\%) & 788 (3.9\%) & \\
\hline$\geq 60,000$ & $82(1.5 \%)$ & $233(1.2 \%)$ & \\
\hline Geography & & & $<0.00 I^{*}$ \\
\hline North & $2,169(40.1 \%)$ & $8,4 \mid 5$ (4I.9\%) & \\
\hline Central & I, I35 (21\%) & $4,129(20.6 \%)$ & \\
\hline South & I,868 (34.5\%) & $7,09 \mid$ (35.3\%) & \\
\hline Other & 235 (4.3\%) & 454 (2.3\%) & \\
\hline Urbanization & & & 0.14 \\
\hline I (highest) & 1,969 (36.4\%) & 7,533 (37.5\%) & \\
\hline 2 & I,376 (25.4\%) & $5,110(25.4 \%)$ & \\
\hline 3 & I,303 (24.1\%) & $4,853(24.2 \%)$ & \\
\hline 4 (lowest) & 759 (I4\%) & 2,593 (I2.9\%) & \\
\hline \multicolumn{4}{|l|}{ Comorbidity } \\
\hline DM & $1,660(30.7 \%)$ & $3,550(17.7 \%)$ & $<0.00 I^{*}$ \\
\hline Hypertension & $2,732(50.5 \%)$ & $6,839(34 \%)$ & $<0.00 I^{*}$ \\
\hline Hyperlipidemia & I,333 (24.7\%) & $4,337(21.6 \%)$ & $<0.00 I^{*}$ \\
\hline CHD & I,533 (28.4\%) & $3,576(17.8 \%)$ & $<0.00 I^{*}$ \\
\hline CKD & 635 (11.7\%) & $\mathrm{I}, 16 \mathrm{I}(5.8 \%)$ & $<0.00 I^{*}$ \\
\hline Chronic liver disease & I,326 (24.5\%) & $3,380(16.8 \%)$ & $<0.001^{*}$ \\
\hline Cerebral vascular accident & I,826 (33.8\%) & $2,755(13.7 \%)$ & $<0.00 I^{*}$ \\
\hline COPD & $2,034(37.6 \%)$ & $3,338(16.6 \%)$ & $<0.00 I^{*}$ \\
\hline
\end{tabular}

Note: aNTD refers to New Taiwan dollars, of which I US dollar $=32$ TWD, ${ }^{*} p<0.05$. Abbreviations: $\mathrm{CHD}$, coronary heart disease; CKD, chronic kidney disease; COPD, chronic obstructive pulmonary disease; DM, diabetes mellitus.

\section{Discussion}

Infection with scabies induces host immune reactions and may increase the risk of subsequent autoimmune diseases. Our study indicates an increased risk of subsequent PA in patients with scabies. These patients have a $51 \%$ higher risk of PA (adjusted HR: 1.51, 95\% CI: 1.09-2.08) than patients who have not had scabies.

PA results in vitamin B12 deficiency and may cause anemia, neuropsychiatric impairment, and osteoporosis in untreated patients. ${ }^{16}$ The long-term risk of gastric cancer is also increased in patients with PA. ${ }^{3,17}$ Therefore, prompt diagnosis of PA and supplementation with vitamin B12 is important. ${ }^{16,18}$ The prevalence of PA is believed to be underestimated due

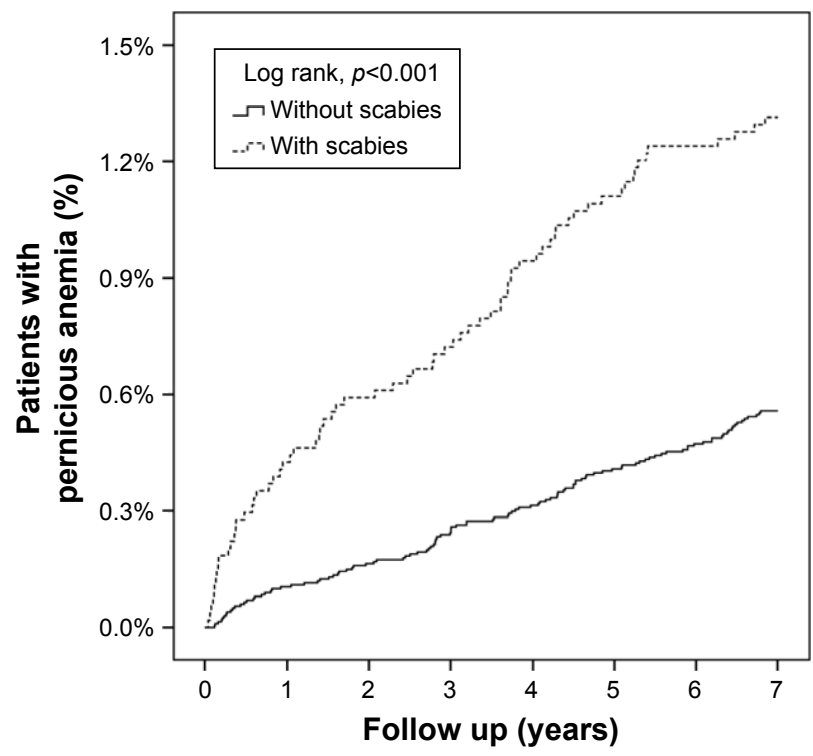

Figure 2 The Kaplan-Meier survival curve for the cumulative incidences of pernicious anemia of both groups is presented $(p<0.00 \mathrm{I})$.

to its nonspecific symptoms and the fact that it is relatively difficult to diagnose. ${ }^{1,16}$ The prevalence of PA varies in different races, ages, diagnostic tools, and countries. For example, the overall prevalence was $1.9 \%$ in Carmel's study, but it was $4.3 \%$ among black women. ${ }^{2}$ The incidence of PA was $0.7 \%$ in the present study. Women and older adults have a higher risk of PA, and our study revealed similar findings. ${ }^{2,19,20}$

The clinical manifestations of PA are vague and nonspecific. Many diseases will cause anemia, and supplementation with iron or folate without investigation is common. PA is easily neglected, and its diagnosis remains a challenge for clinicians. The most important key for the correct diagnosis of PA is clinical suspicion, followed by confirmative laboratory tests. There are several diagnostic modalities for PA, but the convenience, sensitivity, specificity, and accessibility of these are not ideal. ${ }^{21,22}$ Histologically proven atrophic body gastritis via endoscopy is valuable but invasive. Tests for serum vitamin B12, methylmalonic acid, homocysteine, and pepsinogen lack validation. Anti-parietal cell antibodies and anti-intrinsic factor antibodies can be measured to diagnose PA. Anti-parietal cell antibodies are noted in approximately $90 \%$ of patients with the condition, but are not specific to PA. Anti-intrinsic factor antibodies are more specific, but testing for them is less sensitive $(\sim 60 \%) .{ }^{23}$ Therefore, diagnosis of PA is complex, and underdiagnosis is common. According to the findings of our study, a suspicion of PA should be maintained in patients with scabies and coexisting anemia. Further confirmative tests are warranted, and vitamin B12 supplementation is essential. 
Table 2 Independent predictors of risk factors of pernicious anemia by Cox regression analysis in the 7-year follow-up period

\begin{tabular}{|c|c|c|}
\hline & $\begin{array}{l}\text { Crude HR } \\
(95 \% \mathrm{Cl})\end{array}$ & $\begin{array}{l}\text { Adjusted HR } \\
(95 \% \mathrm{Cl})\end{array}$ \\
\hline Scabies & $2.37(1.76-3.19)^{\ddagger}$ & $1.5 \mathrm{I}(1.09-2.08)^{\dagger}$ \\
\hline \multicolumn{3}{|l|}{ Gender } \\
\hline Female & I & I \\
\hline Male & $0.5 \mathrm{I}(0.38-0.69)^{\ddagger}$ & $0.53(0.39-0.7 I)^{\ddagger}$ \\
\hline \multicolumn{3}{|l|}{ Age } \\
\hline $20-29$ & I & I \\
\hline $30-39$ & $0.83(0.3-2.29)$ & $0.77(0.28-2.13)$ \\
\hline $40-49$ & I.6I (0.7I-3.68) & $1.16(0.5-2.69)$ \\
\hline $50-59$ & $2.39(1.09-5.21)^{\dagger}$ & $1.24(0.55-2.82)$ \\
\hline $60-69$ & $3.64(1.74-7.6 \mathrm{I})^{\dagger}$ & $1.47(0.66-3.27)$ \\
\hline$\geq 70$ & $5.83(3.05-I I .12)^{\ddagger}$ & $2.22(1.07-4.6 \mathrm{I})^{\dagger}$ \\
\hline \multicolumn{3}{|l|}{ Income group NTD } \\
\hline$<20,000$ & I & I \\
\hline $20,000-39,999$ & $0.49(0.26-0.92)^{\dagger}$ & I (0.65-I.53) \\
\hline $40,000-59,999$ & $0.12(0.02-0.86)^{\dagger}$ & $1.08(0.75-1.55)$ \\
\hline$\geq 60,000$ & $0.4(0.06-2.85)$ & I.I $5(0.5 \mathrm{I}-2.56)$ \\
\hline \multicolumn{3}{|l|}{ Geography } \\
\hline North & 1 & \\
\hline Central & I.22 (0.8I-I.84) & $0.65(0.42-1.01)$ \\
\hline South & $1.51(1.08-2.12)^{\dagger}$ & I.II (0.76-I.6I) \\
\hline Other & $\mathrm{I} .77(0.8 \mathrm{I}-3.87)$ & $0.86(0.54-1.36)$ \\
\hline \multicolumn{3}{|l|}{ Urbanization } \\
\hline I (highest) & 1 & I \\
\hline 2 & $0.67(0.43-1.02)$ & $1.05(0.53-2.08)$ \\
\hline 3 & $1.34(0.94-1.9)$ & $0.33(0.05-2.39)$ \\
\hline 4 (lowest) & $1.29(0.84-1.99)$ & $0.97(0.13-7.12)$ \\
\hline \multicolumn{3}{|l|}{ Comorbidity disease } \\
\hline DM & $3.71(2.78-4.96)^{\ddagger}$ & $1.6(1.14-2.24)^{\dagger}$ \\
\hline Hypertension & $3.99(2.91-5.49)^{\ddagger}$ & $1.28(0.84-1.95)$ \\
\hline Hyperlipidemia & $1.98(1.46-2.67)^{\ddagger}$ & I.07 (0.76-I.52) \\
\hline CHD & $3.05(2.27-4.08)^{\ddagger}$ & I.09 (0.78-I.53) \\
\hline CKD & $5.44(3.95-7.49)^{\ddagger}$ & $2.55(1.8-3.6)^{\ddagger}$ \\
\hline Chronic liver disease & $1.66(1.2-2.3)^{\dagger}$ & I.I $17(0.83-1.65)$ \\
\hline Cerebral vascular accident & $3.8 \mathrm{I}(2.85-5.1)^{\ddagger}$ & $1.28(0.9-1.82)$ \\
\hline COPD & $3.49(2.6 I-4.67)^{\ddagger}$ & $1.57(1.12-2.19)^{\dagger}$ \\
\hline
\end{tabular}

Notes: aNTD refers to New Taiwan dollars, of which I US dollar $=32$ TWD; ${ }^{t} p<0.05$ for comparison between patients with two groups, ${ }^{\ddagger} p<0.001$ for comparison between patients with two groups.

Abbreviations: $\mathrm{CHD}$, coronary heart disease; $\mathrm{Cl}$, confidence interval; $\mathrm{CKD}$, chronic kidney disease; COPD, chronic obstructive pulmonary disease; DM, diabetes mellitus; HR, hazard ratio.

PA is an autoimmune disease and is thought to be part of polyglandular autoimmune syndrome type 2 (PAS 2), which includes such disorders as autoimmune thyroid disease, Addison's disease, type $1 \mathrm{DM}$, and vitiligo. In patients with PA, thyroid disease and DM are common comorbidities, suggestive of the autoimmune polyendocrine syndrome. ${ }^{19}$ In the present study, patients with $\mathrm{DM}, \mathrm{CKD}$, and COPD had higher risks of PA (Table 2, adjusted HR 1.6 for DM, 2.55 for $\mathrm{CKD}$, and 1.57 for COPD). Immune-mediated inflammatory processes are believed to play important roles in the abovementioned three diseases. DM is a well-known autoimmune disease, and IL-6 and IL-17 pathways are involved in the pathophysiology of CKD. ${ }^{24-26}$ IL-17 alteration is thought to be a crucial role in patients with COPD. ${ }^{9} \mathrm{PA}$ is strictly regarded as an autoimmune disease. ${ }^{19}$ Autoantibodies against the proton pump, gastric $\mathrm{H}^{+}, \mathrm{K}^{+}$ATPase, which is highly expressed in parietal cells, are involved in the immune mechanism of PA. 22,23,27 IL-21 and tumor necrosis factor-alpha are highly expressed in animals with autoimmune gastritis. ${ }^{28}$ In patients being treated for hepatitis $\mathrm{C}$ with interferon-alpha, PA can be induced. ${ }^{29-31}$ Similarly, immune-mediated inflammatory processes also play an important role in scabies. Marked cytokine alterations have been noted in scabies. ${ }^{7,8}$ In porcine models, IL-17-related pathways may contribute to the immunopathology of crusted scabies. ${ }^{7}$ In human skin equivalents, multiple host-parasite interactions were revealed. ${ }^{32}$ All of these findings support the possible role of scabies in the immune-mediated nature underpinning the pathophysiology of PA. However, the entire mechanisms of immune-mediated diseases are complex, and it is difficult to elucidate the influences of scabies of these diseases. We found that patients with scabies had a higher risk of subsequent PA, and immune-mediated inflammatory processes may contribute to this relationship. Further studies are warranted to investigate the association and test this hypothesis.

Scabies can be treated with drugs including topical permethrin, lindane, benzyl benzoate, and oral ivermectin. However, lindane, a neurotoxin that interferes with gamma aminobutyric acid neurotransmitter function, may cause aplastic anemia. ${ }^{33}$ Therefore, the World Health Organization has not recommended lindane use since 1995, and lindane is no longer available in the market. Hematological adverse effects have not been reported for other drugs.

The strength of our study is the large study population. In addition, the NHIRD covers approximately $99 \%$ of the 23 million residents of Taiwan, making the analysis broadly representative. However, this study is subject to several limitations. First, the causal relationship between scabies and PA could not be identified directly due to its crosssectional design. Second, details of the laboratory tests are not available in the NHIRD; therefore, a further comparison of laboratory data could not be undertaken; because of this, the severity of anemia and related vitamin B12 and autoantibody levels were unknown. Third, the NHIRD is organized by the ICD-9-CM; therefore, some patients with PA may have been classified as simply having anemia, and the incidence of PA may have been underestimated. The fear 
of unmeasured/imperfectly measured confounders is also a limitation of this study.

\section{Conclusion}

In conclusion, this nationwide population-based study provides evidence indicating a possible relationship between scabies and PA. Specifically, patients with scabies had an increased risk of subsequent PA, with an adjusted HR of 1.51. Immune-mediated inflammatory processes may contribute to this relationship. Further studies are warranted to investigate the entire pathological mechanisms between these two diseases. Physicians should pay attention to patients with history of scabies presented with anemia. Further confirmative tests of pernicious anemia may contribute to correct diagnosis and initiation of vitamin B12 supplement.

\section{Acknowledgments}

The work was supported by grants from the Ministry of Science and Technology Taiwan (MOST 104-2320-B-016012-MY3) and Taoyuan General Hospital, Ministry of Health and Welfare (PYT10601) in the analysis and interpretation of data. Our manuscript has been edited for English language, grammar, punctuation, and spelling by Enago, the editing brand of Crimson Interactive Pvt., Ltd. The current implications and conclusions do not represent the opinions of the Bureau of National Health Insurance, the Department of Health, or the National Health Research Institute.

\section{Author contributions}

All authors made substantial contributions to conception and design, acquisition of data, or analysis and interpretation of data; took part in drafting the article or revising it critically for important intellectual content; gave final approval of the version to be published; and agree to be accountable for all aspects of the work.

\section{Disclosure}

The authors report no conflicts of interest in this work.

\section{References}

1. Toh BH, van Driel IR, Gleeson PA. Pernicious anemia. $N$ Engl J Med. 1997;337(20):1441-1448.

2. Carmel R. Prevalence of undiagnosed pernicious anemia in the elderly. Arch Intern Med. 1996;156(10):1097-1100.

3. Murphy G, Dawsey SM, Engels EA, et al. Cancer risk after pernicious anemia in the US elderly population. Clin Gastroenterol Hepatol. 2015; 13(13):2282-2289.e1-e4.

4. Romani L, Steer AC, Whitfeld MJ, Kaldor JM. Prevalence of scabies and impetigo worldwide: a systematic review. Lancet Infect Dis. 2015; 15(8):960-967.

5. Chosidow O. Scabies. N Engl J Med. 2006;354(16):1718-1727.
6. Chung SD, Wang KH, Huang CC, Lin HC. Scabies increased the risk of chronic kidney disease: a 5-year follow-up study. J Eur Acad Dermatol Venereol. 2014;28(3):286-292.

7. Mounsey KE, Murray HC, Bielefeldt-Ohmann H, et al. Prospective study in a porcine model of sarcoptes scabiei indicates the association of Th2 and Th17 pathways with the clinical severity of scabies. PLoS Negl Trop Dis. 2015;9(3):e0003498.

8. Walton SF, Oprescu FI. Immunology of scabies and translational outcomes: identifying the missing links. Curr Opin Infect Dis. 2013; 26(2):116-122.

9. Chen JY, Liu JM, Chang FW, et al. Scabies increased the risk and severity of COPD: a nationwide population-based study. Int J Chron Obstruct Pulmon Dis. 2016;11:2171-2178. eCollection 2016.

10. Liu JM, Chiu FH, Lin CY, Chang FW, Hsu RJ. Incidence of autoimmune diseases in patients with scabies: a nationwide population-based study in Taiwan. Rheumatol Int. 2017;37(7):1125-1134.

11. Bhat N, Rawat AK. Health status of siblings of hospitalized children. Indian J Pediatr. 2004;71(5):401-403.

12. Cheng TM. Reflections on the 20th anniversary of Taiwan's singlepayer National Health Insurance System. Health Aff (Millwood). 2015; 34(3):502-510.

13. Lin SK, Liu JM, Chang YH, et al. Increased risk of endotracheal intubation and heart failure following acute myocardial infarction in patients with urolithiasis: a nationwide population-based study. Ther Clin Risk Manag. 2017;13:245-253.

14. Chang FW, Lee WY, Liu YP, et al. The relationship between economic conditions and postpartum depression in Taiwan: a nationwide population-based study. J Affect Disord. 2016;204:174-179.

15. ICD-9-CM coding and reporting official guidelines. American Hospital Association, American Medical Record Association, Health Care Financing Administration, National Center for Health Statistics. J Am Med Rec Assoc. 1990;61(Suppl 10):1-17.

16. Stabler SP. Vitamin B12 deficiency. N Engl J Med. 2013;368:149-160.

17. Boysen T, Friborg J, Stribolt K, et al. Epstein-Barr virus-associated gastric carcinoma among patients with pernicious anemia. Int J Cancer. 2011;129(11):2756-2760.

18. Rojas Hernandez CM, Oo TH. Advances in mechanisms, diagnosis, and treatment of pernicious anemia. Discov Med. 2015;19(104):159-168.

19. Hershko C, Ronson A, Souroujon M, Maschler I, Heyd J, Patz J. Variable hematologic presentation of autoimmune gastritis: age-related progression from iron deficiency to cobalamin depletion. Blood. 2006; 107(4):1673-1679.

20. Weck MN, Brenner H. Prevalence of chronic atrophic gastritis in different parts of the world. Cancer Epidemiol Biomarkers Prev. 2006; 15(6): 1083-1094.

21. Bizzaro N, Antico A. Diagnosis and classification of pernicious anemia. Autoimmun Rev. 2014;13(4-5):565-568.

22. Toh BH. Pathophysiology and laboratory diagnosis of pernicious anemia. Immunol Res. 2016;65(1):326-330.

23. Neumann WL, Coss E, Rugge M, Genta RM. Autoimmune atrophic gastritis - pathogenesis, pathology and management. Nat Rev Gastroenterol Hepatol. 2013;10(9):529-541.

24. Davidson A, Diamond B. Autoimmune diseases. N Engl J Med. 2001; 345(5):340-350.

25. Su H, Lei CT, Zhang C. Interleukin-6 signaling pathway and its role in kidney disease: an update. Front Immunol. 2017;8:405.

26. Cortvrindt C, Speeckaert R, Moerman A, Delanghe JR, Speeckaert MM. The role of interleukin-17A in the pathogenesis of kidney diseases. Pathology. 2017;49(3):247-258.

27. Alderuccio F, Sentry JW, Marshall AC, Biondo M, Toh BH. Animal models of human disease: experimental autoimmune gastritis - a model for autoimmune gastritis and pernicious anemia. Clin Immunol. 2002; 102(1):48-58.

28. Nishiura $\mathrm{H}$, Iwamoto $\mathrm{S}$, Kido $\mathrm{M}$, et al. Interleukin-21 and tumor necrosis factor- $\alpha$ are critical for the development of autoimmune gastritis in mice. J Gastroenterol Hepatol. 2013;28(6):982-991.

29. Willson RA. Interferon alfa-induced pernicious anemia in chronic hepatitis C infection. J Clin Gastroenterol. 2001;33(5):426-427. 
30. Andrès E, Loukili NH, Ben Abdelghani M, Noel E. Pernicious anemia associated with interferon-alpha therapy and chronic hepatitis $\mathrm{C}$ infection. J Clin Gastroenterol. 2004;38(4):382.

31. Musialik J, Petelenz M, Błoñska-Fajfrowska B, Hartleb M. Pernicious anemia during peginterferon-alpha2b plus ribavirin therapy for chronic hepatitis C. Eur J Gastroenterol Hepatol. 2009;21(5):593-594.
32. Morgan MS, Arlian LG. Response of human skin equivalents to Sarcoptes scabiei. J Med Entomol. 2010;47(5):877-883.

33. Rauch AE, Kowalsky SF, Lesar TS, Sauerbier GA, Burkart PT, Scharfman WB. Lindane (Kwell)-induced aplastic anemia. Arch Intern Med. 1990;150(11):2393-2395.

\section{Publish your work in this journal}

Therapeutics and Clinical Risk Management is an international, peerreviewed journal of clinical therapeutics and risk management, focusing on concise rapid reporting of clinical studies in all therapeutic areas, outcomes, safety, and programs for the effective, safe, and sustained use of medicines. This journal is indexed on PubMed Central, CAS,
EMBase, Scopus and the Elsevier Bibliographic databases. The manuscript management system is completely online and includes a very quick and fair peer-review system, which is all easy to use. Visit http://www.dovepress.com/testimonials.php to read real quotes from published authors.

Submit your manuscript here: http://www.dovepress.com/therapeutics-and-clinical-risk-management-journal 